\title{
Hormone
} Research

ACTH resistance 19

Adrenal insufficiency 19

Adult GH deficiency 35

Androgens 44

Appetite regulation 93

Behavior 55

Blood pressure 38

Body composition 13

- mass index 59

- proportions 38

Bone metabolism 13

- mineral density 38

Calcineurin 24

Carbohydrate metabolism 13, 38

Cardiac effects 38

Cardiovascular diseases 44

Clinical practice 98

Computer network 59

Congenital adrenal hyperplasia 19

Cortisol 1

$\mathrm{CrescNet}^{\circledR} 59$

c-ret oncogene 67

Crohn disease 13

Cushing disease 19

Deficiency 86

\section{Elderly 51}

Endochondral ossification 7

Endocrinology 98

Epiphyseal growth plate 7

Estrogens 82

Euthyroidism 74

Final height 38,44

Generic or disease/study specific 51 Genes 44
Ghrelin 93

Glucocorticoids 7

Glucocorticosteroids 13

Glucose metabolism 44

Glutamine synthetase 24

Growth 13, 19, 59

- disorders 59

- hormone $1,7,13,24,44,55,93$

- - deficiency 29

- _ dosing 35

- - receptor 93

- - replacement 35

- - secretagogue 93

- - therapy 29

- - treatment 38

Hormone replacement 51

$11 \beta$-Hydroxysteroid dehydrogenase 1

Hypertension 44

Hypertrophic chondrocyte differentiation 7

Hypogonadism 86

Hypothyroidism 74

IMAGe association 19

Inflammatory bowel disease 13

Insulin resistance 44

Insulin-like growth factor binding protein-3 29

- _ factor-I 7, 13, 24, 29, 44

Ischaemic heart disease 44

Linear growth 7

Menopause 82

Morbidity 44

Mortality 44

Multiple endocrine neoplasia 67

Obesity 1,59

Octanoic acid 93
Oestrogens 38, 44

Outcome 55

Peptide modification 93

Population 59

Prediction models 29

- of future 98

Premature menopause 82

Progestogens 82

Protein metabolism 13

Psychiatric symptoms 55

Puberty 44, 82

Quality of life 55

_ $\quad$ -, health-related 51

Replacement 86

- therapy 74

Screening 59

Steroid myopathy 24

Stomach 93

Testosterone 86

Thyroid hormone 7

Thyrotropin 74

Thyroxine 74

Triiodothyronine 74

Tumour suppressor 67

Turner syndrome 38, 44

Tyrosine kinase receptor 67

Weight 59 